\title{
EL PAPEL DE LA DOCENCIA UNIVERSITARIA EN LA FORMACIÓN INICIAL DE PROFESORES
}

\section{Introducción}

En este artículo presentamos algunos hallazgos logrados en la investigación "Acción y Saber Pedagógico en la Formación Inicial Docente", investigación realizada a partir de la experiencia en cursos de formación inicial de profesores de la Facultad de Educación de la Universidad Alberto Hurtado, UAH.

La motivación de llevar a cabo este estudio nace de suponer que, para formar profesores que desarrollen una labor educativa en las escuelas, que contribuyan a que sus alumnos logren aprendizajes que les permitan insertarse en las distintas dimensiones de la vida (social, ciudadana, laboral, económica, entre otras), es necesario que se formen en lógicas inclusivas que les posibiliten pensarse en su desempeño profesional. Ello requiere, a nuestro entender, poner atención a las prácticas de quienes los formamos, de manera que los futuros profesores construyan un saber pedagógico para realizar acciones docentes al servicio de sus alumnos, dándoles a estos últimos la oportunidad de prepararse para enfrentar el mundo.

\section{La problemática}

En nuestro país el desempeño de los profesores de las escuelas y liceos es objeto permanente de cuestionamiento por parte de la sociedad en su conjunto, pues parece no satisfacer las demandas del contexto educativo y social actual. Esto se evidencia cuando se sabe de problemáticas complejas que se viven al interior de los establecimientos escolares, tales como la violencia entre alumnos o de ellos hacia sus profesores, o cuando se conocen los magros resultados de la prueba SIMCE o la Prueba de Selección Universitaria (PSU) o que los mismos profesores obtienen en las evaluaciones docentes o incluso antes de ejercer en la prueba INICIA. Esto pone el ejercicio 
del profesor en tela de juicio, y con ello, su formación, lo que desafía a las escuelas de pedagogía a revisar su propuesta didáctica, puesto que la formación inicial explica la calidad del quehacer del profesor (Fuentealba, 2003).

Diversos estudios, tanto en Chile como en América Latina, han observado que en la formación inicial existen problemáticas tales como la desconexión entre la teoría y la práctica, fragmentación del currículum de formación, falta de articulación entre las instituciones formadoras y las escuelas y desconexión entre las materias y su pedagogía (Vaillant, 2009; Mineduc, 2005; Pogré, 2004). La existencia de estas debilidades desatiende las imágenes, creencias y valoraciones con que llegan los futuros profesores al inicio de su formación, dejándolas sin modificación una vez terminado el trayecto educativo, lo que resulta preocupante si consideramos que las prácticas pedagógicas se basan en estas creencias (Latorre, 2004).

Ante estas debilidades, la Escuela de Educación de la UAH se ha propuesto enfrentar algunas de las problemáticas de la formación docente inicial y ofrecer un modelo educativo que favorezca en el futuro profesor un desarrollo profesional identitario y un modo particular de vincularse con la institución escolar. Para ello, imparte un bachillerato durante los dos primeros años de carrera y un sistema de educación dual en el que la formación pedagógica se realiza tanto en la universidad como en la escuela, buscando de esta manera vincular a los estudiantes con la realidad concreta, ofrecerles un referente experiencial y fomentar su capacidad reflexiva ${ }^{2}$.

En este contexto, para que los alumnos puedan enfrentar la complejidad del ámbito escolar, participan en talleres que vinculan el saber universitario con el experiencial, logrado una vez inmersos en las escuelas. El Taller de Introducción a la Práctica Educativa (TIPE) prepara a los estudiantes para la formación dual, y el Taller de Reflexión de la Práctica Educativa (TRPE) acompaña el comienzo de

2 Para una descripción exhaustiva de la propuesta formativa, consultar el documento preparado por los directores de las carreras de pedagogía de la Facultad y publicado por el CIDE; en http://www.cide.cl/Download/Saber_\%20pedagogico.pdf y en http://mt.educarchile.cl/MT/ Falvarez/2006/09/saber_pedagogico_y_formacion_d.html 
dicha formación. Los cursos que se consideraron para efectos de este estudio corresponden a estas asignaturas, cuyas propuestas se orientan a incentivar la participación y el permanente cuestionamiento por parte de los estudiantes para desarrollar sus habilidades reflexivas.

La problemática que da origen a este estudio nace de la positiva respuesta de los estudiantes a las propuestas pedagógicas de estos talleres. Ella se expresa en una participación que da cuenta de actitudes y discursos que transitan, a través de los cursos, de lo anecdótico a lo teórico, de lo descriptivo a lo reflexivo, de lo discreto a lo expresivo, de naturalizar a problematizar la realidad.

Ante esta respuesta, nos preguntamos: ¿cuál es la contribución de la acción pedagógica llevada a cabo en estos cursos a la construcción del saber docente de los estudiantes? ¿Qué aprenden, acerca de lo que es ser profesor, a partir de dicha acción que observan y experimentan como estudiantes? Desde estas interrogantes, se plantea como objetivo principal de esta investigación describir la contribución de la acción pedagógica llevada a cabo en los cursos TIPE y TRPE a la construcción del saber docente de los futuros profesores de inglés de la UAH. Así, describir la acción pedagógica y conocer el saber construido por los estudiantes como resultado de esta acción surgen como objetivos específicos de esta investigación.

\section{Importancia de la investigación}

Esta investigación es importante por varias razones. La primera es porque hay un escaso número de investigaciones que describan los procesos formativos y los problematicen para encontrar pistas que orienten la mejora de la formación inicial. Estas mejoras docentes se discuten en el ámbito de políticas educativas, es decir, atendiendo principalmente los niveles macro y meso del currículum, quedando fuera el nivel micro, que desde la perspectiva planteada aquí es fundamental para explicar la calidad de los procesos formativos. También es importante porque, a pesar de que existen estudios que abordan la formación inicial docente, hay pocos que consideren las voces de los propios estudiantes que participan de ella como un aporte clave para el mejoramiento de sus experiencias formativas. Esta 
investigación es relevante, además, porque considera desarrollar una práctica pedagógica a partir de un saber propio, que comprende los alcances de los procesos que promueve y fortalece el ejercicio docente porque permite construcción de saber desde la propia práctica. Como afirma Quirós (1989), generalmente la docencia y la investigación van por caminos separados, estando esta última generalmente asociada a la especialidad profesional en lugar de la práctica educativa, por lo que considerar la propia práctica pedagógica como objeto de estudio es fundamental para fortalecer el ejercicio docente.

\section{Referentes para el estudio}

En cuanto a los aspectos contextuales, este estudio nace a partir de la experiencia docente en los Talleres TIPE y TRPE en la carrera de Pedagogía en Inglés de la UAH, cuya propuesta formativa enfatiza la formación de estudiantes críticos y comprometidos con la justicia social, aspiración que concuerda con el espíritu jesuita que define la misión de la universidad.

En términos teóricos, en este estudio se entiende la formación inicial docente vinculada al concepto de currículum, por lo que comprende el conjunto de vivencias que recorren los alumnos en el trayecto formativo, principalmente en dos niveles. En un nivel meso, refiere a las vivencias de los estudiantes, definidas por condiciones institucionales en que se enmarca la formación, las que se definen por aspectos tangibles -infraestructura y tiempos para la socialización y/o actividades transversales o complementarias para la educacióncomo intangibles -calidad de las relaciones que median en distintos espacios de la institución o selección de asignaturas que componen la malla curricular-.En el nivel micro, el currículum en la formación inicial se relaciona con las prácticas docentes, es decir, por la acción pedagógica llevada a cabo por quienes forman a los futuros profesores. Esto se expresa en la manera que tienen los docentes de materializar procesos pedagógicos de enseñanza-aprendizaje y contempla la relación pedagógica que construye ese docente; la selección cultural que hace para la asignatura; racionalidad con la que se relaciona al saber; así como a los procesos de organización, transmisión y evaluación de dicha selección. En otras palabras, para efectos de este 
estudio entenderemos el currículum de formación inicial como una práctica cultural (Da Silva, 1998), es decir, como un trayecto en el cual se van conformando identidades, las que comportan visiones de mundo que son adquiridas por quienes viven ese recorrido.

La importancia de lo que se señala anteriormente radica en posicionar la acción pedagógica como aspecto crucial del currículum de formación, entendiéndola como una acción cultural, que nos remite a prácticas que transmiten formas de entender el mundo y relacionarse. De esta forma, es el docente quien, desde sus modos de ser y hacer, transmite estas comprensiones a los estudiantes para que las incorporen en sus propios contextos de referencia. De ahí la importancia de revisar ese hacer, de explicitar su intención, de hacerse cargo de él y de tensionar colectivamente su finalidad. Así visto, la acción pedagógica será entendida como una acción intencionada que realiza el docente, mediante un proceso creativo y comunicativo, de negociación y diálogo, que se sitúa en un grupo humano con el carácter de una trama viva, compleja, incierta, que está siempre inacabada, abierta a su revisión y, por lo tanto, a su recreación.

La concepción de acción pedagógica que se plantea implica que el contenido del currículum no extrae su significación desde objetivos predeterminados, sino de reflexiones de quienes están implicados en la acción -docentes y estudiantes- quienes adquieren protagonismo por igual en la encarnación de él desde la reflexión (Bobadilla, Cabrera y Cárdenas, 2003). Esta acción se traduce así en una didáctica cuyo elemento central es la interacción, llevada a cabo como un proceso de construcción de significados por parte de los participantes.

El concepto de acción pedagógica al que se remite este estudio se encarna en un practicum reflexivo, es decir, que se sitúa en la relación con los sujetos y sus subjetividades, en un intento inacabado e inacabable de comprenderlas y así construir colectivamente un espacio de negociación de significados, que implica una acción comunicativa que redundará en una creación y recreación permanente de la cultura y de los propios sujetos. Para comprender este planteamiento, es necesario discutir qué entenderemos entonces por reflexión, por diálogo y por aprendizaje. 
En primer término, la reflexión se entiende desde una racionalidad comunicativa, es decir, como un ejercicio de explicar los supuestos e ideas preconcebidas que subyacen en la actividad práctica y en valorar las consecuencias que derivan de la acción (Van Manem, 1977). Por otra parte, el diálogo es un requisito de la reflexión, que se expresa no sólo como mero intercambio informativo, sino también como un intercambio dialéctico, que redunda en un cuestionar las premisas sobre el saber, sobre sí mismo y el mundo (Brockbank y McGill, 2002). Por último, el concepto de aprendizaje ha sido abordado como "un proceso de búsqueda personal y social, a través del cual se aprende a ser, actuar, interactuar y aprender" (Echeverría, 2009). Este concepto es importante porque se espera que desde esta acción pedagógica los estudiantes construyan saber.

La discusión sobre el concepto de saber pedagógico es extensa. Para Latorre (2002), es un saber "en uso", inmanente en la acción, es decir, oculto a los ojos del profesor. Este se construye en la praxis (Barrera, 2009; Mena y Romagnoli, 1993), y está formado por representaciones a partir de las cuales los profesores orientan su acción (Tardif, 2004). Ello significa entender que el saber no es objetivo ni está fuera del sujeto, sino que en él, en su experiencia (Messina, 2008). Considerando que la experiencia puede aprehenderse no sólo desde el intelecto sino también desde los sentidos, se entiende además que este saber no tiene sólo una dimensión cognitiva, sino también una afectiva/sensitiva. En suma, es importante que los profesores desarrollen habilidades reflexivas para que así construyan un saber pedagógico que les permita orientar sus prácticas en coherencia con su intencionalidad.

Por otra parte, las representaciones que conforman el saber pedagógico son elaboradas por los sujetos a lo largo de su vida escolar. Si se considera que son ellas las que orientarán la acción del futuro profesor, resulta fundamental que la formación inicial las aborde intencionalmente, pues operan como verdaderos esquemas de pensamiento, de acción e interacción (Bobadilla, Cárdenas, Dobbs, Soto, 2009). Estas creencias en que se sustentan las prácticas pedagógicas son previas a la entrada a los programas de formación docente inicial y son difícilmente modificadas por éstos (Latorre, 
2002), lo que resulta preocupante si consideramos que basado en estas representaciones el futuro profesor orienta su actuar. Es por ello que recuperarlas para tensionarlas y someterlas a su crítica es fundamental en la formación inicial.

\section{Decisiones metodológicas para el estudio}

El enfoque de investigación adoptado para este estudio se enmarca en el paradigma cualitativo interpretativo, y la metodología que se ha elegido es estudio de caso, en tanto el foco de atención se centra en la particularidad de la experiencia vivida en los cursos TIPE y TRPE desde una acción pedagógica cuya propuesta es singular.

Los sujetos del estudio de caso son los estudiantes de la carrera de Pedagogía en Inglés de la UAH que han cursado los cursos TIPE y TRPE en los años 2008 y 2009, que asistieron regularmente a clases y que se interesaron y tuvieron disponibilidad para participar en los grupos de discusión que se convocaron para efectos de este análisis. Esta experiencia es reconstruida desde la observación de las clases, la percepción que los estudiantes tienen de ellas y el saber mismo que evidencian desde distintos productos creados con fines evaluativos. Para esto, los instrumentos de recogida de datos definidos son los registros de observación de clase, los grupos de discusión y el análisis documental de productos creados por los estudiantes durante el semestre que fueron sometidos a evaluación. El procedimiento para realizar el estudio de caso consistió en recopilar recursos para su análisis, y seleccionarlos en igual número para cada curso de acuerdo a su relevancia. El estudio se realizó finalmente con 12 registros de observación de sesiones de clase, la transcripción de 4 grupos de conversación y 12 creaciones estudiantiles.

En la fase de análisis de las evidencias la pesquisa se realizó instrumento por instrumento. Para discutir los hallazgos encontrados, se reordenó la información obtenida de acuerdo a los objetivos del estudio, lo que significó una descripción de la acción pedagógica a partir de la información recogida en los registros de observación y los discursos, y en una descripción del saber construido por los estudiantes a partir de la información recogida en los registros de 
observación, en las sesiones de discusión y en las creaciones de los estudiantes.

Los criterios de rigor para llevar a cabo la investigación fueron la triangulación de distintos datos y la metodológica, el juicio crítico de pares, la descripción detallada de la experiencia y la confirmación de registros consultando a los participantes.

En cuanto a los resguardos éticos, esta investigación se basa en el interés por contribuir a la mejora de un problema, es decir, a generar un beneficio social, desde un trabajo riguroso que da fe de la validez de lo recogido para hacer el estudio. También es de interés ético el que esta investigación favorezca las condiciones de un diálogo auténtico, y que cuente con el consentimiento informado por parte de los sujetos participantes, resguardando su identidad y la de quienes son mencionados en las sesiones de los grupos de discusión.

\section{Hallazgos del estudio}

Las evidencias recogidas desde los registros de observación, las creaciones estudiantiles y los grupos de discusión permiten describir la acción pedagógica, el saber construido por los estudiantes, los obstaculizadores y facilitadores de la propuesta para la construcción del saber y la valoración que los alumnos hacen de la experiencia. Lo que este artículo se propone es dar cuenta brevemente de parte de los hallazgos, específicamente los relacionados con la descripción de la acción pedagógica, por medio de la cual se identificaron varias características, todas ellas con estrechas relaciones entre sí. Entre otros rasgos, se puede señalar que la acción pedagógica es ética, rupturista, democrática, dialógica, solidaria, transformadora, flexible y gradual. No obstante lo anterior, las dos que se mencionan en primer lugar son fundamentales pues constituyen un punto de partida a las demás, posibilitado por la decisión del profesor de plantear las finalidades de su acción y de orientarla por una concepción de aprendizaje en tanto proceso de búsqueda personal y social. Así visto, el carácter ético se describe a través de categorías que emergieron en el análisis y que nos remiten a que la acción pedagógica explicita intenciones, fomenta la autenticidad, aspira a la distribución equitativa del poder, 
es consecuente, ejemplificadora y respetuosa de los sujetos. En otras palabras, la acción da cuenta de un sentido ético porque se preocupa de las consecuencias que tiene sobre los demás sujetos. Una estudiante señalaba al respecto en un grupo de discusión:

"Y...aparte que no es como que te diga "esto es así y es así". (Sino que) Es como: "no, poh, chiquillos, a ver, qué piensan ustedes". Entonces son cosas como que te transmite la honestidad pa' que tú también seai' honesto... (...) porque hay otras clases en donde tú tení que ser cínica".

En cuanto a la concepción de enseñanza-aprendizaje como práctica rupturista, de significación y de subjetivación, tenemos una acción pedagógica que posibilita concretar la intención ética señalada anteriormente al canalizarla en una propuesta de enseñanzaaprendizaje que implica la conciencia, la experiencia y la subjetividad. Que sea una práctica de significación se refiere a que invoca los sentimientos y/o la experiencia personal, como recurso didáctico. Los contenidos no son el eje de la clase sino que la complementan, se fomenta la producción de sentido y se vinculan con la experiencia personal. Por otro lado, que esta acción conlleve una evaluación que constituya una práctica de subjetivación se traduce en que fomenta la creatividad, es exigente, y evalúa de forma no tradicional el proceso de subjetivación de los estudiantes. Por último, que sea una práctica rupturista se refiere a que genera un insight en los estudiantes, despierta interés, dispone favorablemente y provoca reflexión. En síntesis, la clave de esta concepción de enseñanza-aprendizaje radica en que las cosas adquieran sentido para aquel que se quiere que aprenda, y ello requiere una vinculación con el sujeto, con su subjetividad y con su experiencia, con lo que a éste le pasa, de manera que esté presente, es decir, que se involucre su conciencia en el proceso. Un estudiante lo expresa en un grupo de discusión:

"Es como "yo no te voy a poner lo que tú tienes que hacer sino (...) pone una pregunta, "ipero tú por qué piensas que es así?" Entonces ahí uno empieza como a reflexionar, "sí, por qué hice esto". "iAh! por esto" y empiezas a pensar y empiezas a estar más consciente de las cosas". 
Otro estudiante también lo manifiesta cuando plantea:

"Sí, como que el cerebro está partido y piensa un poco... el mío no hacía eso (risas). Estaba dormido, entonces como que empezai' de a poquito, en las horas de clase, ya, piensa un poquito, piensa otro poco, y piensa otro poco y después empezai' a pensar en tu casa, y en la micro..."

Tanto el sentido ético de la acción como la concepción de enseñanza-aprendizaje son rasgos fundamentales para construir una relación pedagógica en que el poder se distribuya de manera más democrática en el aula, favoreciendo un clima solidario. Que la acción sea democrática se refiere a que el docente no se arroga para sí el derecho de tomar decisiones sin considerar las posturas de los estudiantes. Por el contrario, se entiende como una forma de plantearse en el aula que, sin renunciar a la autoridad, entiende el poder intrínseco a su papel como un poder hacer cosas para el crecimiento y desarrollo de las personas a través de una convivencia democrática (Prieto, 1998, en Prieto, 2001). Esta característica se sustenta en que la acción promueve la autonomía, permite participar, considera la voluntad de los sujetos y escucha sus posturas. El siguiente fragmento de un registro de observación ilustra este rasgo de la acción pedagógica:

Una vez que la sala quedó en silencio, la profesora, apuntando a la diapositiva, comenzó diciendo: "Bueno, supongo que se acuerdan que de acuerdo al cronograma hoy nos corresponde comenzar esta unidad, que ya tenía unas lecturas definidas... de acuerdo a lo que conversamos la clase pasada, vamos a dejar esta sesión para definir qué tensiones quieren ustedes discutir, y así entre todos podamos elegir las lecturas para abordar esos temas y la forma en que lo trabajaremos...".

El rasgo democrático y democratizador de la acción pedagógica redunda en la dialogicidad en el aula, lo que fomenta el desarrollo de espacios de interacción y diálogo, además de la participación voluntaria. Los alumnos se expresan; el profesor escucha con atención y, respetuosamente, recoge lo que dicen buscando su profundización; los estudiantes dialogan unos con 
otros y en esta lógica el docente distribuye su tiempo para atenderlos a todos. Las evidencias recogidas muestran que esta acción también abre espacios para la participación y la interacción, buscando el entendimiento entre los sujetos. Aprender es algo que va más allá de los contenidos y es que los destinatarios se involucran afectivamente en el proceso. Todo lo anterior quiere decir que la acción pedagógica intenciona un encuentro entre los sujetos, lo que es posible al promover métodos de interacción para la construcción de sentidos que redunden en aprendizajes auténticos.

La profesora anotó los temas sobre la diapositiva que tenía los espacios en blanco junto a cada fecha de las clases disponibles. Luego, le pidió al curso que decidieran cómo querían trabajar esos textos, si se harían fichas, si se leerían en clase o si se les ocurría otra forma de hacerlo. Nuevamente, entre todos opinando e interrumpiéndose, se decidió que no se harían más fichas, que la profesora llevaría los textos a la clase, que serían textos breves, y se leerían en clase. La profesora añadió que se trabajarían leyéndolos en grupo, y haciendo un mapa conceptual breve sobre esa lectura para, al momento de abrir la discusión, cada grupo se apoyara en dicho mapa para contribuir a la discusión con conceptos.

A medida que se desarrollan relaciones dialógicas y es la comprensión del otro lo que regula la interacción, se genera un clima de aula solidaria, otro rasgo de la acción pedagógica que emerge del análisis. Las evidencias dieron cuenta de categorías que configuran este rasgo, indicando que la acción pedagógica se vale de un lenguaje de cercanía; favorece la construcción de una intimidad en el aula; genera un entusiasmo en los sujetos; fomenta el involucramiento afectivo, además de despertar el interés, compromiso y buena disposición por parte de los alumnos. Como expresa una estudiante al respecto,

"ayudó para relacionarnos y para crear nuevos espacios para hablar, entonces acá fui feliz, creo, durante todo el semestre".

El clima de aula solidario resulta fundamental para disponer favorablemente la participación de los estudiantes y con ello 
su transformación, cuestión que emerge como otra importante característica. Ésta se expresa en un cambio en la manera de ver las cosas, en actitudes, en concepciones y en sus valoraciones. Un estudiante da cuenta de esto cuando expresa:

"Yo ahora puedo... eh... prescindir de ciertas cosas como obligatorias en un profesor, yo puedo, como le decía, prescindir de gritar, prescindir de sentarme, de pararme delante de todos al frente. Puedo prescindir de casi todas las cosas que uno conoce como las prácticas de un profesor porque ahora me di cuenta de que hay otras cosas, que uno puede hacer que las cosas se pueden ver de otra manera y que también funciona".

La transformación de los estudiantes redunda en un círculo virtuoso que facilita la generación de la dialogicidad y permite que las características centrales de la acción se vean fortalecidas. En la medida que lo que sucede en el aula sea educativo, es decir, en que desarrolle la conciencia y la autonomía de los sujetos, éstos se transforman, lo que favorece, aún más, que la distribución del poder se democratice. Todo este ciclo está atravesado por dos rasgos adicionales que caracterizan la acción pedagógica: la gradualidad y la flexibilidad. Esto significa que ella genera cambios graduales en el aula, sutiles en un principio, más nítidos después, los que no se dan en todos los sujetos al mismo tiempo ni en un mismo grado ni forma, y que se adaptan a las necesidades emergentes de la clase. Es importante tener en cuenta esto, sobre todo si consideramos que los estudiantes observan rasgos que dificultan una acción dialógica, en tanto han vivido un proceso de escolarización que les ha restado autonomía, autenticidad para relacionarse en el aula y ánimo de participación genuina. Aun cuando estos rasgos condicionan la propuesta formativa, no debieran determinarla, pues de ser así se estaría desconociendo la posibilidad de que la educación se constituya en un proceso de transformación de los sujetos. Esta sí es posible desde una propuesta de formación dialógica, pues en ella se implica la conciencia del sujeto, como queda evidenciado en el siguiente fragmento de un grupo de discusión:

"Yo antes no hablaba. Y mire ahora (risas generales) No, pero es verdad. Este es un tema ya sabido por los 
chiquillos, todo...yo antes les decía todo a las chiquillas al oído..."yo pienso esto..."...pero llegaba el día de la clase y nos sentábamos en círculo... para mí era un terror estar ahí al frente suyo y que me moría porque me dijera "usted qué piensa". Entonces pa' mí eso era una cosa terrorífica".

Por lo tanto, los aspectos centrales de una acción pedagógica, que se plantee como educativa, son la distribución del poder en el aula y la concepción de enseñanza-aprendizaje orientada a un constructivismo social y basada en la dialogicidad de la propuesta didáctica. Son centrales porque queda al arbitrio del docente decidir sobre ellos y encarnarlos en sus prácticas pedagógicas. No distribuir el poder ni establecer la dialogicidad como eje de la relación de enseñanza/aprendizaje limita la clase a un proceso de transmisión de conocimientos, en el cual se ignora si éstos están siendo efectivamente compartidos y/o significados por los estudiantes, y en el que, de esta forma, se refuerza el papel pasivo de los futuros profesores. Éstos cuando limiten a sus propios alumnos reproducirán un círculo vicioso en el que no se logra generar aprendizajes significativos, pues no ha habido un involucramiento genuino en el proceso de enseñanza.

\section{Conclusiones}

Es importante considerar la incidencia de la acción pedagógica en los procesos formativos, sobre todo cuando se refiere a la formación de profesores, pues en ella no sólo hay una enseñanza de contenidos -independientemente del campo de estudio al que correspondan-sino que sobre todo hay un aprendizaje, no necesariamente consciente, de una forma de entender el papel del docente en el aula, el cual es transmitido desde dicha acción y que el futuro profesor incorpora a sus contextos de referencia.

Que dicha acción pedagógica intencione la tensión y emergencia de esos contextos que el sujeto ha construido a través de su vida escolar, permitirá que puedan ser modificados en su formación si se hacen emerger esas creencias para ser sometidas a la revisión del mismo individuo. Esto requiere de una acción pedagógica que se 
entienda como un encuentro, un diálogo intersubjetivo desde el cual el futuro profesor logre hacerse consciente de sus creencias, vaya descubriéndose y configurando su identidad, cuestión que requiere, en primer término, que sienta que puede plantearse auténticamente.

La acción pedagógica de estudio fue logrando esto en la medida que se planteó como ética, al cautelar las consecuencias de la propuesta en los sujetos y al estar orientada por una concepción de enseñanza-aprendizaje basada en un constructivismo social, dando lugar así a una relación pedagógica más horizontal, sometida a la crítica de los participantes del proceso; y al promover la participación auténtica de los estudiantes, es decir, sin hacer uso de motivaciones extrínsecas. Asimismo, la acción pedagógica se mostró favorecedora de un clima de aula en el que los estudiantes no se sintieran presionados o atemorizados por participar; orientada a la transformación de los sujetos en términos de que incorporaran las nuevas comprensiones sobre el mundo que el diálogo les ayudaba a levantar; gradual y flexible, es decir, respetuosa de los tiempos de los estudiantes, atendiendo que cada individuo es único y singular, por lo que no es posible esperar que el proceso educativo se viva como homogeneizador.

Rescatar la importancia de la acción pedagógica es atender al nivel micro del currículum, del que poco se discute cuando de la formación de profesores y de calidad de educación se trata. Es sabido que mejorar esta enseñanza es fundamental, como también lo es revisar el concepto de calidad. Aludir a éste, como tan insistentemente se hace en la actualidad, a veces sólo implica generar consenso sobre su necesidad, desatendiendo una discusión profunda del término en sí, que permita aclarar qué categorías se están poniendo en juego cuando se aborda el concepto.

Aun cuando se tuvieran políticas educativas que expliciten una formación inicial de calidad en términos de mallas curriculares, o en otros términos macro o institucionales, no se puede desconocer que el currículum se encarna en prácticas pedagógicas concretas que posibilitan o dificultan que los futuros profesores se apropien del saber, razón por la cual es esencial entender que, tanto o más importante que discutir sobre la calidad de la formación inicial docente, es destacar 
la relevancia de la acción pedagógica de los formadores de profesores como factor clave por el que pasa dicha calidad. Lo anterior se explica dado que, finalmente, es en la forma en la que nos relacionamos que la educación se constituye en proceso que atiende a la individuación y a la socialización de los sujetos. Hablar de calidad en el proceso formativo es, entonces, hablar sobre la condición de las relaciones en que se basa esa enseñanza. Entenderlo así releva el papel de las prácticas pedagógicas de los formadores de profesores en la calidad de su educación.

\section{Referencias bibliográficas}

Barrera, F. (2009) Desarrollo del profesorado: el saber pedagógico y la tradición del profesor como profesional reflexivo. En Acción Pedagógica n. ${ }^{\circ}$ 18, enero-diciembre, 2009, pp. 42-51. En: http://www.saber.ula.ve/ bitstream/123456789/29541/1/articulo4.pdf

Bobadilla, M., Cárdenas, A., Dobbs, E. y Soto, A.M. (2009) Los rodeos de la práctica. Representaciones sobre el saber docente en el discurso de estudiantes de pedagogía: una propuesta de lectura del saber docente. Revista Estudios Pedagógicos n. ${ }^{\circ}$ 35, Estudios Pedagógicos XXXV , n. ${ }^{\circ} 1$ : pp. 239-252, 2009.

En: http://mingaonline.uach.cl/pdf/estped/v35n1/art14.pdf

Bobadilla, M., Cárdenas, A. y Cabrera, I. (2003) Hacia un nuevo sentido de la evaluación. Una experiencia didáctica en la formación docente inicial. En Revista Electrónica Diálogos Educativos. Año 03, n. ${ }^{\circ}$ 5, 2003. En: http://www.umce.cl/ dialogos/n05_2003/bobadilla.swf

Brockbank, A. y McGill, I. (2002) Aprendizaje reflexivo en la educación superior. Madrid, Morata.

Da Silva, T. (1998) Currículum y cultura como práctica de significación. En Revista de Estudios del Currículum, Vol. 1, n. ${ }^{\circ}$ 1. Barcelona, PomaresCorredor.

Echeverría, P. (2009) ¿Qué significa aprender para los estudiantes secundarios? Sus sentidos y comprensiones en una experiencia de aprendizaje por proyecto. Revista Electrónica Diálogos Educativos. Año 8, n. ${ }^{\circ}$ 16, 2008. En: http://www.umce.cl/ dialogos/n16_2009/echeverria.swf

Fuentealba, R. (2003) La inserción profesional de profesores principiantes de enseñanza media del área humanista de la Región Metropolitana. Tesis de doctorado, Facultad de Educación Pontificia Universidad Católica de Chile. 
Latorre, M. (2005) Continuidades y rupturas entre formación inicial y ejercicio profesional docente. Revista Iberoamericana de Educación. n. ${ }^{\circ}$ 36/2, 10/06/05).

Latorre, M. (2004) Aportes para el análisis de las racionalidades presentes en las prácticas pedagógicas. Revista Estudios Pedagógicos, Facultad de Filosofía y Humanidades de la Universidad Austral de Chile, n. ${ }^{\circ} 30$, 2004, pp. 75-91.

Latorre, M. (2002) Saber pedagógico en uso: caracterización del saber actuante en las prácticas pedagógicas. Tesis doctoral. Pontificia Universidad Católica de Chile.

Mena, M. y Romagnoli, C. (1993) Convivencia social en la enseñanza media. Santiago, CPU.

Messina, G. (2008) Formación docente: del control al saber pedagógico. Revista Docencia, Colegio de Profesores de Chile, n. ${ }^{\circ}$ 34, mayo 2008, pp. 78-86.

Ministerio de Educación (2005) Informe sobre la Comisión de Formación Inicial Docente. Santiago, Serie Bicentenario.

Pogré, P. (2004) Escuelas que enseñan a pensar. Buenos Aires, Papers Editores.

Prieto, M. (2001) Mejorando la calidad de la educación. Valparaíso, Ediciones Pontificia Universidad Católica de Valparaíso.

Quirós, T. (1989) Docencia e Investigación. Cuaderno de Investigación en la Educación, n. ${ }^{\circ}$ 1. Centro de Investigaciones Educativas. Facultad de Educación, Río Piedras, Universidad de Puerto Rico. En: http://cie. uprrp.edu/cuaderno/ediciones/01/pdfcuadernol/clart6.pdf

Tardif, M. (2004) Los saberes del docente. Madrid, Narcea.

Van Manen, M. (1977) Linking ways of knowing with ways of being practical. Curriculum Inquiry, 6. pp. 205-228.

Vaillant, D. (2009) Formación de profesores de educación secundaria: realidades y discursos. Revista de Educación 350. Septiembre-diciembre 2009. pp. 105-122.

Recibido: 16/04/2010

Aceptado: 20/05/2010 